been solved. It shows quaternary changes, subunit rotations again, and tertiary changes similar to those seen between the $a$ and $b$ forms. Signal transmission results from a change of subunit packing and alterations of the tertiary structure of the $\alpha$ helix linking the subunit interface and the catalytic site. In the $a$ form, the sequence around the phosphorylated serine residue has $\alpha$-helical form, forming new interactions between subunits in the dimer. The effector AMP site is located near the dimer interface, far from the pyridoxal phosphate moiety at the active site.

Changes in substrate affinity are best understood in bacterial phosphofructokinase $^{5}$, a tetrameric enzyme in which effectors bind close to subunit interfaces and to the substrate site of a neighbouring subunit (see figure). The differences between the two allosteric forms include a remarkable local change: the end of an $\alpha$ helix situated between the effector and substrate sites unfolds, and an arginine side chain is replaced by glutamic acid. Because, in the $\mathrm{R}$ form, the arginine interacts with the phosphate group of the substrate fructose 6-phosphate, the affinity for the substrate falls dramatically. Classical kinetic studies of $E$. coli phosphofructokinase implying a very large change of affinity between the $\mathrm{R}$ and $\mathrm{T}$ forms are nicely vindicated by this new structure.

The activity of mammalian phosphofructokinases is regulated by associationdissociation rather than by conformation changes alone ( $\mathrm{J}$. Lee, University of Missouri, St Louis). This mechanism is easily reconciled with allostery. In haemoglobin, subunit dissociation is also coupled to substrate binding, although this has no physiological function in mammals. Careful measurements of the dissociation constant yield values of the relative free energy of ligated forms with between one and four ligands. The results suggest that molecules with two bound ligands may represent a third allosteric form (G. Ackers, Johns Hopkins University), possibly in agreement with direct measurements of the relative abundance of the various ligated forms by cryoscopic methods (M. Perella, University of Milan).

While cooperativity can be accommodated in the original model $^{2}$, anti-cooperativity cannot be, but is instead the basis of the model of Koshland et al. ${ }^{6}$. Its structural interpretation has now emerged from the X-ray study of glyceraldehyde 3phosphate dehydrogenase from Bacillus stearothermophilus?. High-resolution structures for the tetramer and for several complexes with the coenzyme NAD were described by A. Wonacott (Imperial College, London). In the free enzyme, the subunits are identical and have dihedral symmetry. In a newly determined structure with one NAD per tetramer, the symmetry is lost because of alterations of tertiary structure induced by ligation.
Further changes of the same nature cannot easily be accommodated in other subunits unless the quaternary structure also changes, which it does in the fully ligated protein, restoring dihedral symmetry.

While the interactions of bacterial repressor proteins with DNA may not be part of allostery in the strict sense, they certainly belong to a Jacques Monod conference. Examples were presented of the $\operatorname{trp}$ repressor $^{8}(\mathrm{P}$. Sigler, University of Chicago) and the met repressor (S. Phillips, University of Leeds). Though unrelated, both proteins are dimers, they carry the characteristic helix-turn-helix motif for DNA binding and the co-repressor site is near the interface with DNA. The corepressor, tryptophan in one case and Sadenosyl-methionine in the other, appears to make direct contacts to phosphate groups on DNA. Its binding induces conformation changes in trp, but not in met.

The possibility of direct interaction between ligands as an alternative to allostery was raised ${ }^{1}$ in 1963 , and may be the basis for the non-covalent regulation of another classical regulatory enzyme, $E$. coli glutamine synthetase. X-ray analysis reveals that tryptophan and histidine, two of a score of nitrogen metabolites among its inhibitors, bind close to the active site and may overlap with the substrate (D. Eisenberg, University of California, Los Angeles). But covalent regulation is more efficient than non-covalent inhibition (E. Stadtman, National Institutes of Health) and the dramatic effects of adenylation of tyrosine residues far from active sites, still to be explained at the atomic level, are not unlike those induced by effectors in allosteric enzymes.

While the scope of allostery may be less than its original authors hoped, structural data on haemoglobin and several allosteric enzymes fit the theory remarkably well, given that so little was known in 1965. Effector sites, subunit movements and symmetry are consistently observed, and their thermodynamic coupling explains cooperativity and regulation. Equilibrium binding studies and kinetics may be more difficult to reconcile with the simplicity of the equations of the model of Monod et al., yet it remains the best we have - if only for its cartesian elegance.

Joël Janin is Professor of biophysics at Université Paris-Sud, Orsay and director of the Laboratoire de Biologie Physicochimique, 91405-Orsay, France.

\footnotetext{
1. Monod, J., Changeux, J.P. \& Jacob, F. J molec Biol 6 306 (1963)

2. Monod, J. Wyman, J. \& Changeux, J.P. J. molec. Biol. 12 88 (1965)

3. Kantrowitz, E.R. \& Lipscomb, W.N. Science 241, 669 (1988)

4. Sprang, S.R. et al. Nature 336. 215 (1988)

5. Shirakihara, Y. \& Evans, P.R. J. molec. Biol. 204, 973 (1988)

6. Koshland, D.E. Jr, Neméthy, G. \& Filmer, D. Biochemistry 5, 365 (1966).

7. Skarzynski, T. \& Wonacott, A.J. J. molec. Biol. 203, 1097 (1988)
}

8. Otwinowski, Z, et al. Nature 335, 321-328 (1988)

\section{Sky snatcher}

Television is a massive time-waster. One can skim rapidly through a book, a journal, or a newspaper to see if anything appeals; but television demands real-time attention. It seldom repays it. Even a dedicated channel hopper can waste hours every day in a tedious hunt for worthwhile snippets of entertainment. Now Daedalus automates that hunt.

DREADCO's 'Snatcher' is a combined video recorder and pattern-recognition computer. It receives all channels simultaneously, and records any topic or type of image that it has been programmed to watch out for. At daily or weekly intervals, its owner can play back the tape: a surrealistic succession of clips and snatches encapsulating everything he would have found interesting for the whole period. All the boredom of trawling the air waves in real time is avoided.

The main problem is how to tell the Snatcher what to watch for. The audio channel is the simplest indicator; the telephone-tapping industry has already developed very effective computer algorithms for identifying specific voices or words in context. Those who want every utterance of Mrs Thatcher, or every reference to reinforced concrete, will be quite easy to satisfy. But a visual interest, perhaps in horses or figure-skating or bedroom scenes, will test current patternrecognition techniques to the limit. The viewer will have to provide a coded set of visual cues or patterns for the Snatcher to watch for, and optimize them until its 'catch' is satisfactory.

For those households which keep the TV switched on all the time, but look at it only occasionally, Daedalus has a more fundamental approach. He plans to equip the Snatcher with a viewing sensor. It fits on the TV set, and scans the room with an infrared beam, seeking the characteristic front-surface reflection returned by eyes looking at the set. An 'artificial intelligence' learning algorithm will thus be able to discover which types of scene are actually watched. Over the weeks it will refine a complex, inscrutable, but increasingly reliable criterion of viewability. Soon the Snatcher will be able to watch on its own, recording everything of household interest.

Vast amounts of time will thus be reclaimed for reality. Most TV is really watched negatively - thus we watch the news to reassure ourselves that nothing very important has happened. The Snatcher will extend that assurance to the whole broadcase output. Pressure groups like anti-pornographers will also welcome the Snatcher. They could program it to detect every example of risqué viewing, and to send off a computer-generated letter of complaint. No human viewer need risk being corrupted.
David Jones 